\title{
A Quantum Version of Wigner's Transition State Theory
}

Received: 15 December 2008 / Accepted: 8 January 2009 / Published online: 24 February 2009

(C) The Author(s) 2009. This article is published with open access at Springerlink.com

\begin{abstract}
A quantum version of a recent realization of Wigner's transition state theory in phase space is presented. The theory developed builds on a quantum normal form which locally decouples the quantum dynamics near the transition state to any desired order in $\hbar$. This leads to an explicit algorithm to compute cumulative quantum reaction rates and the associated Gamov-Siegert resonances with high accuracy. This algorithm is very efficient since, as opposed to other approaches, it requires no quantum time propagation.
\end{abstract}

\section{Introduction}

High-resolution spectroscopic techniques allow one to study chemical reaction processes in unprecedented detail (see, e.g., the review paper [1]). A reaction can often be viewed as a transition across a saddle point of the potential energy surface which describes the interaction between the constituent atoms. In the 1930s Eyring, Polanyi and Wigner developed transition state theory (TST) which is a computationally efficient way to compute classical reaction rates without integrating trajectories. The main idea is to define a dividing surface that partitions the energy surface into a reactant and a product component and compute the rate from the directional phase space flux through this surface. In order not to overestimate the rate the dividing surface must not be recrossed by reactive trajectories. For two degrees of freedom such a dividing surface was constructed from a periodic orbit in the 1970s by Pechukas, Pollak and others [2,3]. In higher dimensions it has recently been shown that a dividing surface free of local recrossings is spanned by a normally hyperbolic invariant manifold (NHIM) [4]. The NHIM and the dividing surface can be explicitly constructed from an algorithm which computes the (classical) normal form of the system near the saddle and leads to a local nonlinear decoupling of the classical dynamics [5].

Much effort has been devoted to developing a quantum TST which inherits the computational benefits of classical TST. The mere existence of such a theory is discussed controversially in the literature. For example, in [6] Miller states that "...there is no uniquely well defined quantum version of TST in the sense that there is in classical mechanics. This is because tunneling along the reaction coordinate necessarily requires one to solve the (quantum) dynamics for some finite region about the TS dividing surface, and if one does this quantum mechanically there is no theory left, i.e., one has a full dimensional quantum dynamics treatment that is ipso

This article is based on the presentation by H. Waalkens at the Fifth Workshop on Critical Stability, Erice, Sicily.

R. Schubert $\cdot$ H. Waalkens $\cdot$ S. Wiggins

School of Mathematics, University Walk, University of Bristol, Bristol BS8 1TW, UK

E-mail: roman.schubert@bris.ac.uk

E-mail: s.wiggins@bris.ac.uk

H. Waalkens $(\bowtie)$

Department of Mathematics, University of Groningen, Nijenborgh 9, 9747 AG Groningen, The Netherlands

E-mail: h.waalkens@rug.nl 
facto exact, a quantum simulation." In this short note we present a quantum TST based on a quantum version of the normal form procedure which, classically, yields the realization of TST. Similar to the classical case this quantum normal form (QNF) leads to a local decoupling of the quantum dynamics to any desired order in $\hbar$ in terms of elementary operators with well known spectral properties. This supersedes a full quantum simulation. In this approach the cumulative reaction probability can be viewed as the quantum mechanical flux through a (classically recrossing free) dividing surface which inherently includes tunneling. In this sense, our approach completely sidesteps the issues and concerns expressed by Miller. The QNF computation can be implemented as an explicit algorithm which leads to an efficient procedure to compute cumulative reaction rates and the corresponding Gamov-Siegert resonances [7]. For the technical details of this note we refer to [8,9], which also includes the historical background for the development of the QNF theory.

\section{The Quantum Normal Form}

The key idea of our approach is to find a unitary transformation which simplifies the Hamilton operator near the saddle point of the corresponding classical dynamics (for the precise meaning of 'near' and the classicalquantum correspondence see [8,9]). More precisely, suppose that the classical system has an equilibrium point of saddle-center- . - -center stability type ('saddle' for short), i.e., the matrix in the associated linearized classical Hamilton equations of motion has one pair of real eigenvalues $\pm \lambda$ associated with the saddle or "reaction coordinate' and $f-1$ pairs of imaginary eigenvalues $\pm \mathrm{i} \omega_{k}, k=2, \ldots, f$, associated with the center or 'bath' degrees of freedom. Suppose furthermore that the $\omega_{k}$ are rationally independent. Then for each $N \geq 2$, there is a unitary transformation $U_{N}$ such that

$$
U_{N}^{\star} \widehat{H} U_{N}=H_{\mathrm{QNF}}^{(N)}\left(\widehat{I}, \widehat{J}_{2}, \ldots, \widehat{J}_{f}\right)+\widehat{R}^{(N+1)},
$$

where $H_{\mathrm{QNF}}^{(N)}$ is a polynomial of order [N/2] in the elementary operators

$$
\widehat{I}=\frac{\hbar}{\mathrm{i}}\left(q_{1} \frac{\mathrm{d}}{\mathrm{d} q_{1}}+\frac{1}{2}\right), \quad \widehat{J_{k}}=-\frac{\hbar^{2}}{2} \frac{\mathrm{d}^{2}}{\mathrm{~d} q_{k}^{2}}+\frac{1}{2} q_{k}^{2} \quad(k=2, \ldots, f) .
$$

We call $H_{\mathrm{QNF}}^{(N)}\left(\widehat{I}, \widehat{J}_{2}, \ldots, \widehat{J}_{f}\right)$ the QNF of order $N$. The properties of the reaction operator $\widehat{I}$ (which is unitarily equivalent to the inverted harmonic oscillator, see [9]) and the harmonic bath operators $\widehat{J}_{k}$ are explicitly known. Since they also commute we can solve the quantum problem described by $H_{\mathrm{QNF}}^{(N)}\left(\widehat{I}, \widehat{J}_{2}, \ldots, \widehat{J}_{f}\right)$ analytically. The remainder $\widehat{R}^{(N+1)}$ is an operator which is small near the saddle in a semiclassical sense, see [9]. Similar to the classical normal form the QNF and $U_{N}$ can be computed order by order using the explicit algorithm developed in $[8,9]$. We note that if we give up the rational independence of the $\omega_{k}, k=2, \ldots, f$, one can find a unitary transformation which brings the original Hamiltonian into a quantum version of the classical normal form reported in [10]. However, similar to the classical case, the quantum problem can in general not be solved explicitly if the $\omega_{k}$ are rationally dependent.

\section{Cumulative Reaction Probabilities and Quantum Resonances}

The eigenfunctions of $H_{\mathrm{QNF}}^{(N)}\left(\widehat{I}, \widehat{J}_{2}, \ldots, \widehat{J}_{f}\right)$ are products of harmonic oscillator wave functions for the operators $\widehat{J}_{k}, k=2, \ldots, f$, and eigenstates of the operator $\widehat{I}$ which can be chosen to be pairs of incoming or outgoing scattering wavefunctions associated with reactants and products [9]. Relating the pairs of incoming and outgoing states leads to a 'local' $S$-matrix which has a simple block structure with the $2 \times 2$ matrices

$$
S_{n}(E)=\frac{\mathrm{e}^{\mathrm{i}\left(\frac{\pi}{4}-\frac{I}{\hbar} \ln \hbar\right)}}{\sqrt{2 \pi}} \Gamma\left(\frac{1}{2}-\mathrm{i} \frac{I}{\hbar}\right)\left(\begin{array}{cc}
-\mathrm{ie}^{-\frac{\pi}{2} \frac{I}{\hbar}} & \mathrm{e}^{\frac{\pi}{2} \frac{I}{\hbar}} \\
\mathrm{e}^{\frac{\pi}{2} \frac{I}{\hbar}} & -\mathrm{ie}^{-\frac{\pi}{2} \frac{I}{\hbar}}
\end{array}\right)
$$

along the diagonal. Here $n$ denotes the vector $\left(n_{2}, \ldots, n_{f}\right)$ of quantum numbers of the modes in the center directions, and $I(E)$ is implicitly defined by 


$$
H_{\mathrm{QNF}}^{(N)}\left(I, \hbar\left(n_{2}+1 / 2\right), \ldots, \hbar\left(n_{f}+1 / 2\right)\right)=E .
$$

This matrix incorporates the effects of tunneling for energies close to the saddle-energy.

Interestingly, the local S-matrix contains the full information required to compute the cumulative reaction probability $N(E)$ and Gamov-Siegert resonances. In fact, $N(E)$ is given as the sum over the individual transmission probabilities of all modes $n$, i.e.,

$$
N(E)=\sum_{n} T_{n}(E), \quad \text { where } \quad T_{n}(E)=\left|S_{n 12}(E)\right|^{2}=\left(1+\mathrm{e}^{-2 \pi \frac{I}{\hbar}}\right)^{-1} .
$$

Moreover, the poles of $S_{n}(E)$ at $I=-\mathrm{i} \hbar\left(n_{1}+1 / 2\right), n_{1} \in \mathbb{N}_{0}$, give the complex energies of the resonances via (4).

\section{Examples}

In Fig. 1 we compare the cumulative reaction probability and Gamov-Siegert resonances computed from the QNF to the corresponding exact results for a 3-degree-of-freedom system consisting of an Eckart potential $V_{\text {Eckart }}(x)=A \exp \left(x+x_{0}\right) /\left(1+\exp \left(x+x_{0}\right)\right)+B \exp \left(x+x_{0}\right) /\left(1+\exp \left(x+x_{0}\right)\right)^{2}$ with $x_{0}=\ln (B+A) /(B-A)$ $(B>A \geq 0)$ in one degree of freedom, and two Morse oscillators $V_{\text {Morse } ; k}\left(x_{k}\right)=D_{\mathrm{e} ; k}\left(\exp \left(-2 x_{k}\right)-\right.$ $\left.2 \exp \left(-x_{k}\right)\right), k=2,3$, in the other degrees of freedom, plus the kinetic coupling $\epsilon\left(p_{1} p_{2}+p_{1} p_{3}+p_{2} p_{3}\right)$.

In the uncoupled case, $\epsilon=0, N(E)$ increases as a function of $E$ at integer steps each time a new transition channel opens, i.e., when the transmission probability $T_{\left(n_{2}, n_{3}\right)}(E)$ of a mode $\left(n_{2}, n_{3}\right)$ of the two Morse oscillators switches from 0 to 1 . For both the uncoupled and strongly coupled case the resonances form a distorted lattice parametrized by the mode quantum numbers $\left(n_{2}, n_{3}\right)$ in horizontal direction and the quantum number $n_{1}$ in vertical direction. Each string of constant $\left(n_{2}, n_{3}\right)$ is related to one step of $N(E)$. The agreement of the QNF results with the exact results is excellent and this remains the case even for the strongly coupled system.
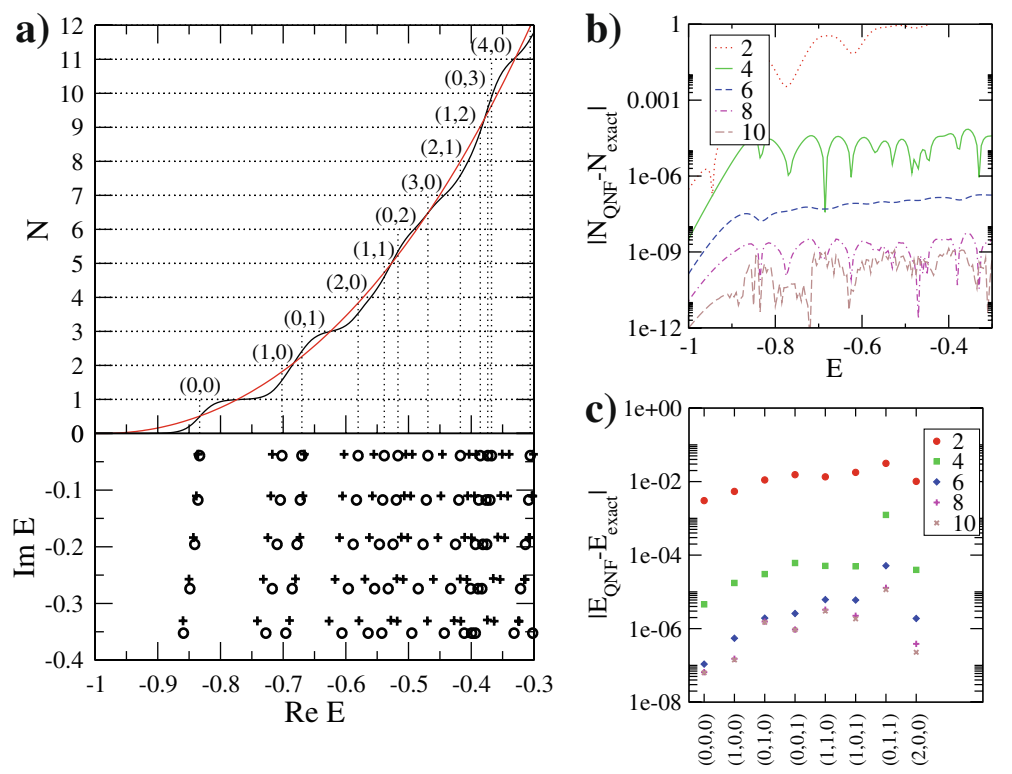

Fig. 1 a The top panel shows $N(E)$ (oscillatory curve) and the classical flux [11] divided by $(2 \pi \hbar)^{2}$ (smooth curve) for the Eckart-Morse-Morse potential defined in the text with $\epsilon=0$. The integers $\left(n_{2}, n_{3}\right)$ mark the energies at which the corresponding Morse oscillator modes contribute a quantization step to $N(E)$. The bottom panel shows the resonances in the complex energy plane marked by circles for the uncoupled case $\epsilon=0$ and by crosses for the strongly coupled case $\epsilon=0.3$. b Errors for the cumulative reaction probability in a for different orders of the QNF. $\mathbf{c}$ Errors for a selection of resonances $\left(n_{1}, n_{2}, n_{3}\right)$ computed from the QNF for $\epsilon=0.3$. (The parameters are $A=B / 10=0.5, D_{e ; 1}=1, D_{e ; 2}=1.5, m=1$, and $\hbar=0.1$ ). 


\section{Conclusions}

We presented a quantum version of TST which is fully in the spirit of classical TST in the sense that it requires no quantum simulation although it gives a full account of the tunneling near the transition state. Our approach is based on a quantum normal form which leads to a local decoupling of the quantum dynamics. The quantum normal form can be cast into an explicit algorithm which leads an efficient way to compute cumulative reaction probabilities and Gamov-Siegert resonances for multi-degree-of-freedom systems, for which other methods are no longer feasible.

Acknowledgments H. Waalkens acknowledges support by EPSRC under grant number EP/E024629/1. S. Wiggins acknowledges the support by the Office of Naval Research Under grant number N00014-01-0769.

Open Access This article is distributed under the terms of the Creative Commons Attribution Noncommercial License which permits any noncommercial use, distribution, and reproduction in any medium, provided the original author(s) and source are credited.

\section{References}

1. Skodje, R.T., Yang, X.: The observation of quantum bottleneck states. Int. Rev. Phys. Chem. 23, 253 (2004)

2. Pechukas, P., McLafferty, F.J.: On transition-state theory and the classical mechanics of collinear collisions. J. Chem. Phys. 58, 1622 (1973)

3. Pechukas, P., Pollak, E.: Transition states, trapped trajectories, and claasical bound states embedded in the continuum. J. Chem. Phys. 69, 1218 (1978)

4. Wiggins, S.: Normally Hyperbolic Invariant Manifolds in Dynamical Systems. Springer, Berlin (1994)

5. Uzer, T., Jaffé, C., Palacián, J., Yanguas, P., Wiggins, S.: The geometry of reaction dynamics. Nonlinearity 15, 957 (2001)

6. Miller, W.H.: Spiers Memorial Lecture. Farad. Discuss. 110, 1 (1998)

7. Friedman, R.S., Truhlar, D.G.: Chemical reaction thresholds are resonances. Chem. Phys. Lett. 183, 539 (1991)

8. Schubert, R., Waalkens, H., Wiggins, S.: Phys. Rev. Lett. 96, 218302 (2006)

9. Waalkens, H., Schubert, R., Wiggins, S.: Efficient computation of transition state resonances and reaction rates from a quantum normal form. Nonlinearity 21, R1 (2008)

10. Wiggins, S., Wiesenfeld, L., Jaffé, C., Uzer, T.: Impenetrable barriers in phase-space. Phys. Rev. Lett. 86, 5478 (2001)

11. Waalkens, H., Wiggins, S.: Direct construction of a dividing surface of minimal flux for multi-degree-of-freedom systems that cannot be recrossed. J. Phys. A 37, L435 (2004) 